\title{
FURAÇÃO DE MATERIAL SANDUÍCHE EMPREGANDO BROCAS COM GEOMETRIAS DIFERENTES: CONSTRUÇÃO DE MODELO MATEMÁTICO PARA PREVISÃO DA FORÇA DE AVANÇO
}

\author{
DRILLING OF SANDWICH MATERIAL USING DRILLS WITH DIFFERENT GEOMETRIES: \\ CONSTRUCTION OF A MATHEMATICAL MODEL TO PREDICT THE FORWARD FORCE
}

\author{
Michele Lisboa SILVEIRA ${ }^{1}$, Bruna Aparecida REZENDE ${ }^{2}$ \\ ${ }^{1}$ michelesilveira1991@ gmail.com, https://orcid.org/0000-0003-3467-9595 \\ 2 brunarezende12@yahoo.com.br, https://orcid.org/0000-0003-1930-2589
}

\author{
A R T I C L E I N F O \\ Article history: \\ Received 2020-07-27 \\ Accepted 2020-09-04 \\ Available online 2020-09-04 \\ palavras-chave \\ Furação \\ Material sanduíche \\ Modelo matemático \\ keywords \\ Drilling \\ Sandwich material \\ Matematical model
}

\begin{abstract}
A B S T R A C T
The sandwich materials are thus named because they combine as properties of two types of materials, acquiring a structure with a core and two outer faces. The present research used a sandwich material with aluminum faces and a polyethylene core. One of the ways to process this material is through machining, more specifically in drilling operations, which was the focus of this research. The reduction of the cutting forces is desired during the drilling operation, thus improving the quality of the holes due to the reduction of vibrations in the system. It can also increase tool life, eliminating costs due to longer tool replacement times. Based on this principle, this research allied statistical knowledge applied to engineering in order to construct mathematical models to predict the behavior of the forward force in the drilling of sandwich material using drills with different geometries, denominated twist drill with two edges, twist drill with three edges and Brad \& Spur. The objective was to develop multiple linear regression equations to identify the influence of cutting speed and feed rate on the thrust force for each of the drills studied. In addition, it was investigated whether the drill geometry impacted behavior that could be modeled by mathematical equations with a certain degree of acceptable confidence.
\end{abstract}

R E S U M O

Os materiais sanduiche são assim denominados pois combinam as propriedades de dois diferentes materiais, adquirindo uma estrutura com um núcleo e duas faces externas. Uma das formas de processar este material é por meio da usinagem, mais especificamente em operações de furação, que foi o foco dessa pesquisa. A redução dos esforços de corte é desejada durante a operação de furação, impactando melhor qualidade dos furos devido a redução das vibrações no sistema. Também pode aumentar a vida útil das ferramentas, eliminando custos devido ao maior tempo para substituição das mesmas. Partindo deste princípio, esta pesquisa aliou conhecimentos estatísticos aplicados à engenharia, a fim de construir modelos matemáticos para prever o comportamento da força de avanço na furação de material sanduiche empregando brocas com geometrias diferentes, denominadas Brad \& Spur, helicoidal com duas arestas e helicoidal com três arestas e. O objetivo foi elaborar equações de regressão linear múltiplas para identificar a influência da velocidade de corte e do avanço sobre a força de avanço para cada uma das brocas estudadas. Além disso, foi investigado se a geometria das brocas impactou em um comportamento que pudesse ser modelado por meio de equações matemáticas, com um determinado grau de confiança aceitável. 


\section{N O M E N C L A T U R A}

$\begin{array}{ll} & \text { coeficientes de regressão } \\ \mathrm{E} & \text { constante associada à equação } \\ f & \text { avanço } \\ \mathrm{F}_{\mathrm{f}} & \text { força de avanço } \\ \mathrm{H}_{0} & \text { hipótese nula } \\ \mathrm{H}_{1} & \text { hipótese alternativa } \\ \mathrm{N} & \text { número de testes } \\ \mathrm{p} & \text { valor } \mathrm{p} \\ \mathrm{R}-\mathrm{sq} & \text { coeficiente de correlação } \\ \mathrm{R}-\mathrm{sq}(\operatorname{adj}) & \text { coeficiente de correlação ajustado } \\ v_{\mathrm{c}} & \text { velocidade de corte } \\ \mathrm{y} & \text { variável de resposta }\end{array}$

\section{INTRODUCẼ̃O}

Os materiais sanduíche são materiais estruturais compostos por duas camadas externas ou faces, que são unidas a um núcleo espesso por meio de um adesivo, e são produzidos com a finalidade de obter as melhores características dos materiais selecionados, para que adquiram propriedades mecânicas específicas, como rigidez, tenacidade e resistência.

A operação de furação é utilizada para a união secundária de materiais sanduíche, devido à necessidade de unir estruturas e conceber um produto final. Brocas helicoidais com diferentes geometrias são usadas para a furação de materiais estruturais, como a Brad \& Spur, helicoidal com duas arestas e helicoidal com três arestas, que foram selecionadas nesta pesquisa.

A aplicação de técnicas estatísticas foi utilizada como um referencial para futuras pesquisas, uma vez que foram desenvolvidas três equações matemáticas para a força de avanço, cada uma para uma broca diferente. De maneira geral, este estudo permitiu conhecimento a respeito dos esforços de corte durante a furação de material sanduíche utilizando brocas com geometrias diferentes. As interpretações foram feitas com base na construção de modelos matemáticos para prever o comportamento das brocas.

\section{REVISÃO BIBLIOGRÁFICA}

\subsection{Furação de materiais sanduíche}

De acordo com a norma DIN 8589 (2003), a furação é definida como uma operação de usinagem com movimento de corte circular, ou seja, com movimento rotativo principal. A ferramenta possui movimento de avanço apenas na direção do seu eixo de rotação, que mantém sua posição em relação à ferramenta e à peça. Diversos estudos têm sido realizados a fim de investigar a influência da geometria das brocas na operação de furação. Albuquerque et al. (2009) utilizaram, na furação de carbono epóxido laminado, quatro tipos de brocas: helicoidal, escalonada, Brad \& Spur e broca tipo punhal. A broca que apresentou os melhores resultados com relação às forças de corte e delaminação foi a Brad \& Spur. Segundo os autores, ela foi originalmente desenvolvida para a furação de madeira. Com a geometria pontiaguda do ponto central de corte, as fibras do material são tensionadas de tal maneira a gerar um corte limpo e uma superfície usinada suave.

Parâmetros de resposta como a força de avanço podem ser avaliados quantitativamente, de modo que menores esforços de corte são desejáveis na operação de usinagem. Pesquisas sobre forças na furação são realizadas a fim de reduzir a potência necessária de corte, evitar danos desnecessários na operação e melhorar a qualidade dos furos. A força de usinagem depende de uma série de fatores, como material da peça, geometria, material e afiação da ferramenta, velocidade de corte, lubrificação, entre outros (FERRARESI, 1977).

\subsection{Análise de regressão linear múltipla}

Uma análise de regressão gera uma equação para descrever a relação estatística entre um ou mais preditores e a variável de resposta para predizer novas observações. A regressão linear geralmente usa o método de estimativa de mínimos quadrados comuns, que resulta na equação minimizando a soma dos resíduos quadrados. Modelos com um preditor são conhecidos como regressão simples (SUPORTE MINITAB, 2016).

Por outro lado, de acordo com Montgomery (2004), alguns problemas de regressão envolvem mais de uma variável independente. Neste caso, pelo menos três variáveis independentes são necessárias. O modelo geral é chamado de análise de regressão linear múltipla e é dado pela Equação 1.

$$
=++\cdots++
$$

onde: y é a variável de resposta, \{\} são coeficientes de regressão, e E a constante associada à equação.

$\mathrm{Na}$ presente pesquisa, buscou-se estabelecer a relação entre a variável de resposta força de avanço, enquanto os preditores foram a velocidade de corte e o avanço. Buscaram-se equações que demonstrassem matematicamente o comportamento da força de avanço com os parâmetros de corte para cada broca.

Os resultados de uma regressão identificam a direção, o tamanho e a significância estatística da relação entre um preditor e a resposta. De acordo com Suporte Minitab (2016), consideram-se os seguintes aspectos para interpretação:

- O sinal de cada coeficiente indica a direção da relação;

- Os coeficientes representam a mudança média na resposta para uma unidade de mudança no preditor, mantendo os outros preditores no modelo constantes;

- O valor p para cada coeficiente testa a hipótese nula de que o coeficiente é igual a zero (sem efeito). Portanto, valores $\mathrm{p}$ menores que 0,05 indicam que o preditor é uma adição significante ao modelo;

- A equação prediz novas observações com valores especificados nos preditores.

\section{MATERIAIS E MÉTODOS}

O material de trabalho consistiu em uma placa de material sanduíche constituída de duas faces externas de alumínio e polietileno de baixa densidade como núcleo. Para a operação de furação foram utilizadas as brocas Brad \& Spur, helicoidal com duas arestas e helicoidal com três arestas, representadas na Figura 1. 
a)

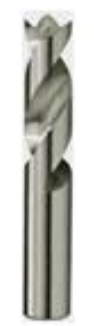

b)

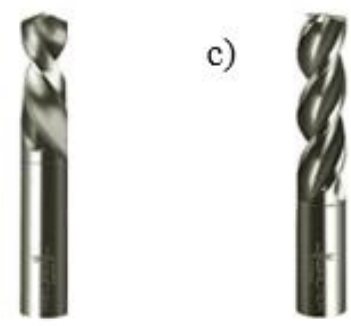

Figura 1 - Brocas utilizadas no experimento a) Brad $\&$ Spur b) helicoidal com duas arestas c) helicoidal com três arestas.

Empregou-se um planejamento fatorial completo, que permitiu o estudo de vários fatores, assim como a interação entre os fatores. Nesta pesquisa, foram considerados como fatores o tipo de broca, a velocidade de corte e o avanço, enquanto os níveis foram as diferentes características ou valores que os fatores possuíam. Os parâmetros utilizados são apresentados na Tabela 1.

Tabela 1 - Parâmetros do experimento.

$\begin{array}{cccccc}\text { Símbolo } & \text { Fatores } & \mathbf{1} & \mathbf{2} & \mathbf{3} & \mathbf{4} \\ & & \text { Brad } & \text { Helic. } & \text { Helic. } & \\ \text { A } & \text { Ferramenta } & \& & \text { com 2 } & \text { com 3 } & - \\ \text { B } & v_{c}(\mathrm{~m} / \mathrm{min}) & 24 & 48 & 72 & - \\ \text { C } & f(\mathrm{~mm} / \mathrm{rev}) & 0,05 & 0,10 & 0,15 & 0,25\end{array}$

\section{RESULTADOS E DISCUSSÃO}

\subsection{Análise de regressão linear múltipla para a força de} avanço

A partir dos resultados gerados para a força de avanço, buscou-se estabelecer uma equação para cada broca relacionando a força de avanço com a velocidade de corte e o avanço. Esta análise é importante pois permite prever o comportamento das brocas quando submetidas a diferentes parâmetros de corte.

Testes foram realizados no Minitab®, Versão 17, a fim de encontrar uma equação com melhores respostas. Inicialmente, realizou-se Análise de Variância para a força de avanço, de acordo com a Tabela 2.

Tabela 2 - Análise de variância para a força de avanço.

\begin{tabular}{|c|c|c|}
\hline $\begin{array}{l}\text { Fonte de } \\
\text { variação }\end{array}$ & Valor $p$ & Contribuiçãa \\
\hline Ferramenta & $\underline{0,000}$ & $76,2 \%$ \\
\hline$v_{c}$ & $\overline{0,000}$ & $7,6 \%$ \\
\hline$f$ & $\overline{0,003}$ & $2,9 \%$ \\
\hline Ferramenta* $v_{c}$ & $\overline{0,442}$ & \\
\hline Ferramenta $* f$ & 0,193 & \\
\hline$v_{c} * f$ & $\underline{0,035}$ & $2,7 \%$ \\
\hline Ferramenta* $v_{c} * f$ & $\begin{array}{c}\overline{0,752} \\
\text { Resumo do modelo }\end{array}$ & \\
\hline $\begin{array}{c}\text { Desvio-padrão } \\
14,0599\end{array}$ & $\begin{array}{c}\text { R-sq } \\
93,52 \%\end{array}$ & R-sq (adj) \\
\hline
\end{tabular}

O planejamento fatorial adequado às condições experimentais foi do tipo $3^{2} 4^{1}$, com dois fatores (velocidade de corte e tipo de broca) em três níveis (três valores para velocidade de corte e três tipos de broca) e um fator (avanço) em quatro níveis (quatro valores de avanço). Houve, portanto, 36 combinações diferentes. Para cada condição de teste realizou-se uma réplica, totalizando 72 experimentos.

Os testes de furação foram realizados no centro de usinagem ROMI, modelo Discovery 560, equipado com comando numérico computadorizado SIEMENS 810D, com potência máxima de $9 \mathrm{~kW}$ e rotação máxima de 7500 rpm.

A célula de carga utilizada possui resolução de $0,01 \mathrm{~N}$, e faixa de operação de 0 a 20 Kgf. É constituída por extensômetros, que sofrem uma deformação quando se aplica uma força sobre ela, fazendo com que ocorra uma diferença de potencial nos terminais de saída da célula de carga. Após aplicada a força, os extensômetros convertem a deformação elástica em variação de resistência elétrica, formando uma ponte de Wheatstone. Esta ponte é uma montagem que serve para encontrar o valor, com boa precisão, de uma resistência elétrica desconhecida.

Utilizou-se um amplificador (LM 358) de $-12 \mathrm{v}$ e $+12 \mathrm{v}$ para amplificar o sinal 100 vezes, ou seja, aumentar a potência do sinal para que a placa de aquisição da National Instruments convertesse-o em um valor digital e o enviasse para o software LabVIEW.

segunda ordem $\left(v_{\mathrm{c}} * f\right)$ apresentaram influência sobre a força de avanço. Como o objetivo foi montar uma equação para cada broca, separadamente, os parâmetros iniciais para montagem das equações seriam $v_{c}, f$, e a interação $\mathrm{v}_{\mathrm{c}} * f$. Assim, o primeiro passo foi elaborar a equação de regressão para a força de avanço com estes três fatores. Porém, as equações geradas não obtiveram uma boa correlação.

Montgomery (2004) destaca que o modelo da Equação 2 é um modelo de regressão linear válido e inclui o polinômio de segundo grau em duas variáveis.

$=++\quad+\quad+$

Onde y é variável de resposta, \{\} são coeficientes de regressão e $\mathrm{X}_{\mathrm{i}}$ são as variáveis associadas.

Com base na Equação 2, verificou-se que, ao elevar ao quadrado as variáveis velocidade de corte e avanço, bem como considerar a multiplicação $v_{c} f$, as equações foram significativas. Portanto, o modelo mais adequado para a força de avanço $\left(\mathrm{F}_{f}\right)$ considerou que esta depende dos seguintes fatores: velocidade de corte $\left(v_{c}\right)$, avanço $(f)$, velocidade de corte $\mathrm{x}$ velocidade de corte $\left(v_{c}^{2}\right)$, avanço $\mathrm{x}$ avanço $\left(f^{2}\right)$, velocidade de corte $\mathrm{x}$ avanço $\left(v_{c} f\right)$, de acordo com a Equação 3 .

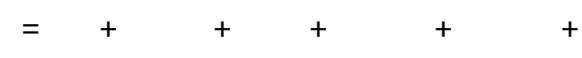

Onde $\mathrm{F}_{\mathrm{f}}$ é a força de avanço, $\{$ \} são coeficientes de regressão, $f$ é o avanço e $v_{c}$ é a velocidade de corte.

Dessa forma, para cada broca, encontrou-se a equação baseada na Equação 3. As equações de regressão encontradas são apresentadas pelas Equações 4,5 e 6. 
Brad \& Spur

3,6

$=19,81+2,02+460-0,024-677-$

Helicoidal com duas arestas

$$
=73,6-0,357+282-0,00135
$$

$-474-1,8$

Helicoidal com três arestas

$$
=127+0,8+421-0,01355^{2}-977^{2}-
$$

1,58

Nas Tabelas 3, 4 e 5, apresentam-se as informações matemáticas relacionadas a cada equação apresentada, e, a partir dos dados, algumas considerações puderam ser feitas. Com relação ao $\mathrm{p}$ valor, para valores menores que 0,05 , pode-se dizer que, para a broca Brad \& Spur, o fator $v_{c}^{2}$ da equação foi o mais influente para o resultado da força de avanço. Para a broca helicoidal com duas arestas, a constante do modelo, $f, f^{2}$ e $v_{c} f$ foram os fatores que mais influenciaram a força de avanço. Para a broca helicoidal com três arestas, a constante da equação foi o que mais aproximou a equação do valor encontrado para a força de avanço.

Tabela 3 - Informações da equação de regressão da broca Brad \& Spur.

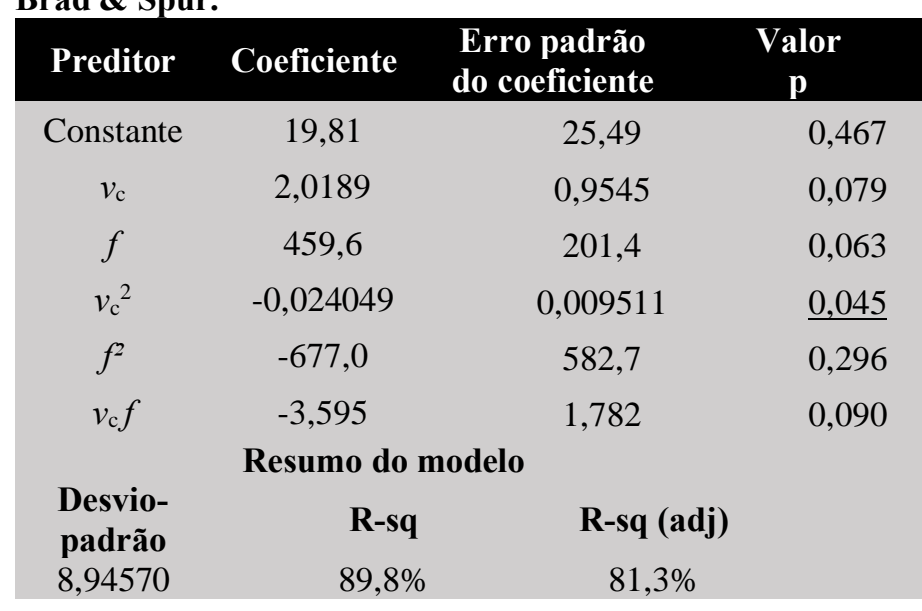

Tabela 4 - Informações da equação de regressão da broca helicoidal com duas arestas

\begin{tabular}{cccc} 
Preditor & Coeficiente & $\begin{array}{c}\text { Erro padrão } \\
\text { do coeficiente }\end{array}$ & $\begin{array}{c}\text { Valor } \\
\text { p }\end{array}$ \\
\hline Constante & 73,648 & 8,407 & $\underline{0,000}$ \\
$v_{c}$ & $-0,3573$ & 0,3148 & 0,300 \\
$f$ & 281,83 & 66,42 & $\underline{0,005}$ \\
$v_{c}{ }^{2}$ & 0,001352 & 0,003137 & 0,681 \\
$f^{2}$ & $-473,6$ & 192,2 & $\underline{0,049}$ \\
$v_{c} f$ & $-1,8049$ & 0,5877 & $\underline{0,022}$ \\
& Resumo do modelo & & \\
Desvio- & R-sq & R-sq (adj) & \\
padrão & 95029 & $96,3 \%$ & $93,1 \%$ \\
\end{tabular}

Tabela 5 - Informações da equação de regressão da broca helicoidal com três arestas

\begin{tabular}{|cccc|}
\hline Preditor & Coeficiente & $\begin{array}{c}\text { Erro padrão } \\
\text { do coeficiente }\end{array}$ & $\begin{array}{c}\text { Valor } \\
\text { p }\end{array}$ \\
\hline Constante & 127,14 & 31,70 & $\underline{0,007}$ \\
$v_{c}$ & 0,798 & 1,187 & 0,527 \\
$f$ & 421,0 & 250,4 & 0,144 \\
$v_{c}^{2}$ & $-0,01350$ & 0,01183 & 0,297 \\
$f^{2}$ & $-976,8$ & 724,6 & 0,226 \\
$v_{c} f$ & $-1,583$ & 2,216 & 0,502 \\
& Resumo do modelo & & \\
Desvio- & R-sq & R-sq (adj) & \\
padrão & $79,9 \%$ & $63,1 \%$ & \\
11,1241 & \multicolumn{3}{c}{} \\
\hline
\end{tabular}

A validade das equações pode ser comprovada pelo Teste de Anderson- Darling, que prevê a normalidade dos dados. A estatística de Anderson-Darling mede o quão bem os dados seguem uma distribuição normal, e ela pode ser utilizada para determinar se os dados atendem à suposição de normalidade para um teste $\mathrm{t}$.

As hipóteses para o teste Anderson-Darling são:

$\AA \mathrm{H}_{0}$ : os dados seguem uma distribuição específica;

$\AA H_{1}$ : os dados não seguem uma distribuição específica;

Deve-se utilizar o valor $\mathrm{p}$ para testar se os dados seguem uma distribuição normal. Se o valor de $\mathrm{p}$ for menor que um alfa escolhido (nesta pesquisa, considerou-se 0,05), a hipótese nula deve ser rejeitada. A Figura 2 apresenta os resíduos correspondentes às equações para cada broca. Os gráficos indicam a validade do modelo com base na análise de $\mathrm{p}$ valor, já que todos foram maiores que 0,05. Portanto, foi aceita a hipótese nula e os dados seguiram uma distribuição normal.

Com relação ao R-sq e R-sq (adj) das equações, apresentados nas Tabelas 3,4 e 5, puderam-se fazer algumas considerações. A equação de regressão para a broca Brad \& Spur apresentou um comportamento intermediário com relação aos modelos das outras duas brocas, ou seja, foi o segundo melhor modelo, com a correlação da equação de $89,8 \%$. Por meio da ANOVA realizada separadamente, a Tabela (6) para a broca Brad \& Spur indicou que apenas a velocidade de corte foi significativa para afetar a força de avanço, e os dados apresentaram uma correlação de 79,23\%.

Analogamente à interpretação para a broca Brad \& Spur, é possível constatar que a broca helicoidal com duas arestas apresentou o melhor modelo para análise de regressão, com uma correlação de 96,3\%. De fato, a Tabela (7), da ANOVA para a broca helicoidal com duas arestas, demonstra que os dados se comportaram com uma correlação de $97,35 \%$, ou seja, a força de avanço depende das condições de velocidade de corte, avanço e interação velocidade de corte * avanço. Os dados da broca helicoidal com duas arestas seguiram um comportamento previsível; logo, sua equação de regressão obteve maior correlação.A broca helicoidal com três arestas foi a que apresentou um modelo de regressão com menor correlação dos dados, e o que mais se aproximou da força de avanço foi o 
coeficiente de ajuste da equação, com um valor de 127,14 . Os dados obtidos para a força de avanço são insensíveis às alterações dos parâmetros de corte, e giram em torno da faixa de 127 N. A análise de variância da broca helicoidal com três arestas (Tabela 8), também revelou a insensibilidade dos dados, mostrando que nenhum fator específico exerceu influência sobre a força de avanço.
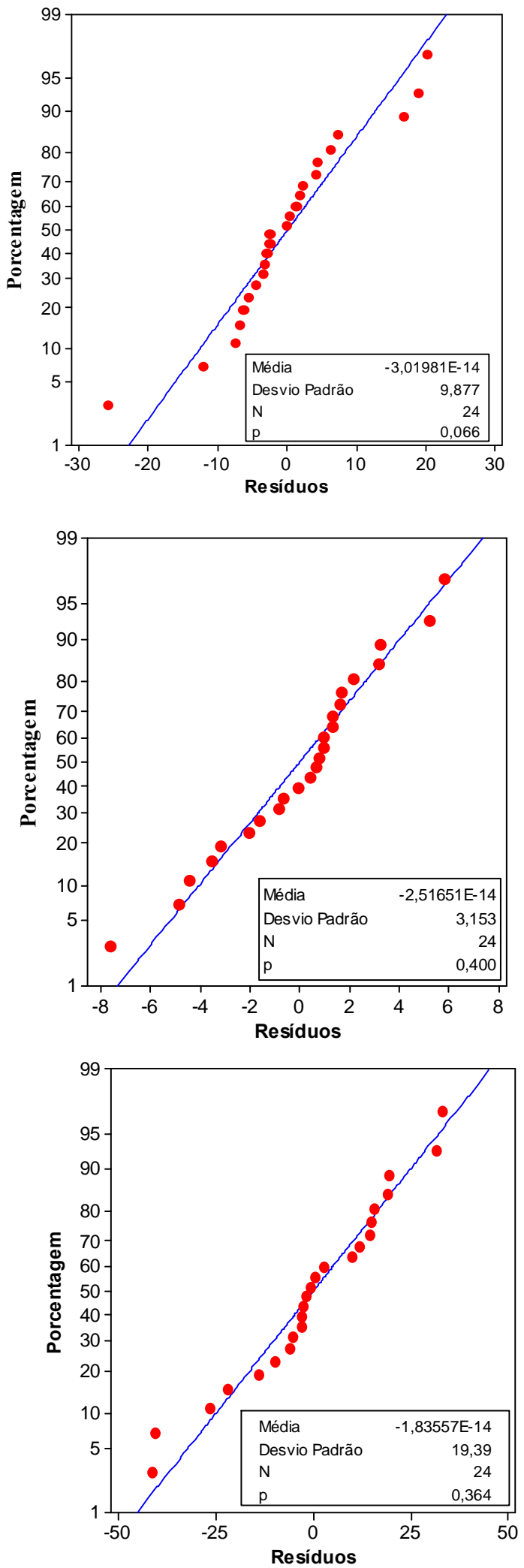

Figura 2 - Análise de resíduos para a equação das brocas a) Brad \& Spur b) helicoidal com duas arestas e c) helicoidal com três arestas.
Tabela 6 - Análise de variância para a broca Brad \& Spur. Fonte de variação $\quad$ Valor p Contribuição

$\begin{array}{ccc}v_{\mathrm{c}} & \underline{0,000} & 60,8 \% \\ f & 0,409 & \\ v_{\mathrm{c}} * f & 0,349 & \\ & \text { Resumo do modelo } & \\ \text {-padrão } & \text { R-sq } & \text { R-sq (adj) } \\ 2412 & 79,23 \% & 60,20 \%\end{array}$

Tabela 7 - Análise de variância para a broca helicoidal com duas arestas.

\begin{tabular}{ccc|} 
Fonte de variação & Valor p & Contribuição \\
\hline$v_{c}$ & $\underline{0,000}$ & $66,2 \%$ \\
$f$ & $\underline{0,000}$ & $22,1 \%$ \\
$v_{\mathrm{c}}{ }^{*} f$ & $\underline{0,003}$ & $8,9 \%$ \\
& Resumo do modelo & \\
Desvio-padrão & R-sq & R-sq (adj) \\
2,56304 & $97,35 \%$ & $94,91 \%$
\end{tabular}

Tabela 8 - Análise de variância para a broca helicoidal com três arestas.

\begin{tabular}{ccc|} 
Fonte de variação & Valor p & Contribuição \\
\hline$v_{\mathrm{c}}$ & 0,153 & \\
$f$ & 0,071 & \\
$\mathbf{v}_{\mathrm{c}} * f$ & 0,344 & \\
\multicolumn{3}{c}{ Resumo do modelo } \\
Desvio-padrão & R-sq & R-sq (adj) \\
20,8965 & $63,71 \%$ & $30,44 \%$
\end{tabular}

4.1 Comparação dos valores medidos e valores previstos

Uma comparação gráfica foi realizada a fim de comparar os valores obtidos no experimento com os valores previstos pela equação, de acordo com as Figuras 3, 4 e 5. Observa-se que a situação em que o valor previsto pelo modelo mais se aproximou do valor real ocorreu para a broca helicoidal com duas arestas.

Figura 3 - Comparação do valor previsto com valor realizado pela broca Brad \& Spur

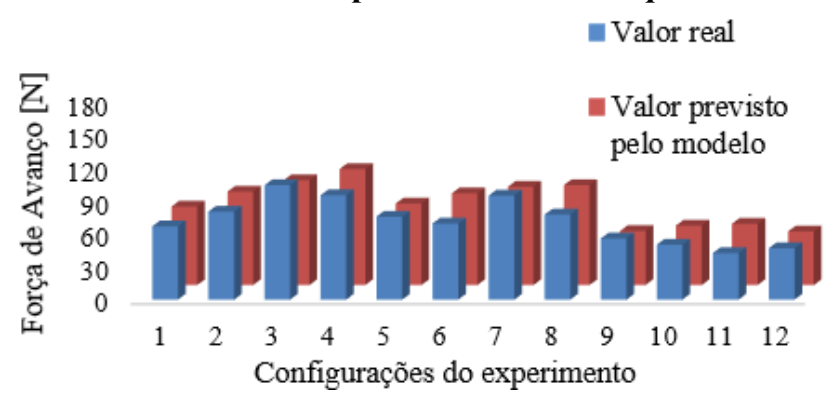


Figura 4 - Comparação do valor previsto com valor realizado pela broca helicoidal com duas arestas

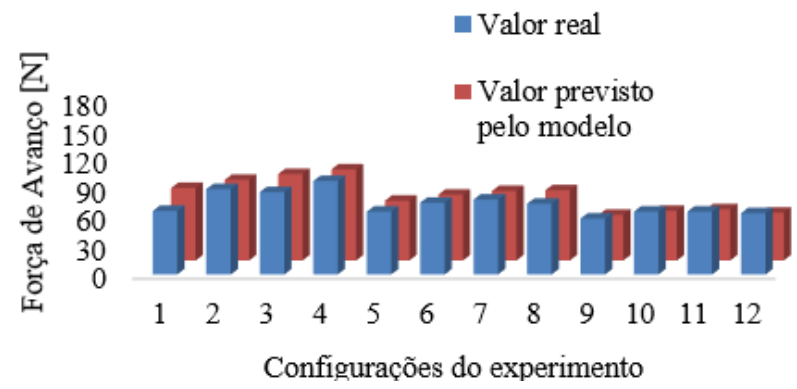

Figura 5 - Comparação do valor previsto com valor realizado pela broca helicoidal com três arestas

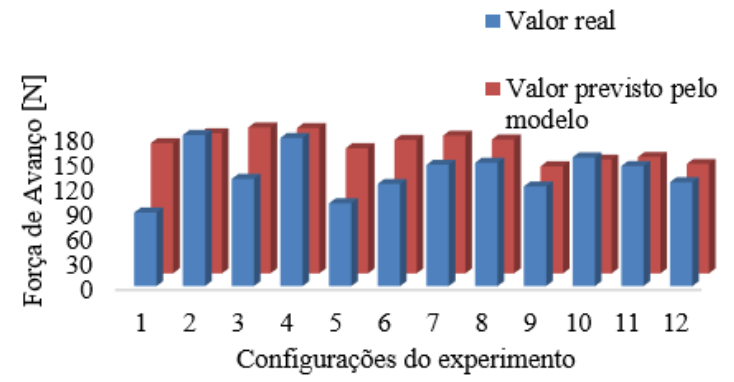

\section{CONCLUSÕES}

A partir dos resultados e discussões apresentados neste trabalho, que tratou da furação do material sanduíche de alumínio e polietileno utilizando brocas com diferentes geometrias e parâmetros de corte, algumas considerações podem ser feitas.

A elaboração das equações de regressão permitiu a observação do comportamento individual da força de avanço para cada broca quando submetida a diferentes condições de corte. A equação obtida para a broca helicoidal com duas arestas foi a que obteve a maior correlação dos dados $(96,3 \%)$, indicando que a velocidade de corte e o avanço exercem influência na variável resposta (força de avanço). Já a broca helicoidal com três arestas apresentou uma equação para a força de avanço insensível aos parâmetros de corte, uma vez que a força de avanço para esta broca se aproximou de um valor constante $127 \mathrm{~N}$.

\section{AGRADECIMENTOS}

Os autores dessa pesquisa agradecem a assistência dos departamentos de Engenharia de Produção e Engenharia Mecânica da Universidade Federal de Minas Gerais, pela disponibilização do Laboratório de Usinagem e Automação. Agradecem às agências de fomento Capes, CNPQ e Fapemig.

R E F E R ÊN C I A S

ALBUQUERQUE, V. H. C. et al. Evaluation of Delamination Damages on Composite Plates using Techniques of Image Processing and Analysis and a Backpropagation Artificial Neural Network. Journal of Composite Materials. v.17, n. 5, p. 120-128, 2009.

DIN 8589 (2003). Manufacturing processes chip removal. Editora Beuth GmbH, 2003.
FERRARESI, D. Fundamentos da Usinagem dos Metais. São Paulo: Edgard Blucher, 1977, 754 p.

MONTGOMERY, D.C. Design and Analysis of Experiments. New York: John Wiley; 2004.

STEMMER, C. E. Ferramentas de corte I. $5^{\text {a }}$ Edição, Florianópolis: Editora UFSC, 2001, 249 p.

SUPORTE MINITAB. Tipos de análise de regressão. Disponível em: <https://support.minitab.com>. Acesso em outubro de 2017. 